\title{
Tres
}

\section{El papel de las \\ universidades en la sociedad 5.0}

DOI: 10.29236/sistemas.n154a9

Reflexión sobre el concepto de Sociedad 5.0 como un componente fundamental de los planes de desarrollo científicos y tecnológicos trazados por el gobierno japonés en los últimos años.

\section{Resumen}

A pesar de que el concepto obedece a condiciones coyunturales muy particulares de la sociedad japonesa, es posible encontrar similitudes en nuestra sociedad colombiana. Sin embargo, la inequidad que caracteriza a nuestro país, requiere de un esfuerzo más articulado entre distintos sectores y tipo de actores. Las universidades, además de reforzar su papel de generadoras de conocimiento, deben reflexionar sobre la pertinencia de los mismos en nuestros contextos y ante nuestras necesidades concretas. La articulación Universidad-Empresa- Estado y la formación a diferentes niveles es un paso importante para la apropiación de los beneficios de la tecnología como se propone en el concepto de la Sociedad 5.0.

\section{Palabras claves}

Transformación, sociedad, universidades, educación. 


\section{Introducción}

La importancia de implementar estrategias de desarrollo sostenibles e inclusivas se concreta en el plan de ciencia y tecnología trazado por Japón en el año 2016 y que introduce por primera vez el término de Sociedad 5.0 (GP, 2016). El término surge como respuesta al ritmo acelerado del desarrollo tecnológico que, en ocasiones, resulta en empresas, comunidades y personas rezagadas que no cuentan con las competencias o recursos necesarios para mantenerse siempre al día sobre las implicaciones transformativas que acarrean estos cambios. Después de la popularización del concepto de la cuarta revolución industrial y de la Industria 4.0, el gobierno japonés, a través de su estrategia de política pública, vuelca la atención de sus planes de desarrollo económico y social en el ser humano, en el individuo. Este planteamiento lleva a un proceso de transformación social que hoy se centra en los desarrollos tecnológicos y en el servicio que la tecnología presta a la sociedad.

La propuesta japonesa tiene como propósito convertirse en un marco social encaminado al diseño e implementación de programas que "garanticen que cada ciudadano pueda florecer y alcanzar una vida próspera", (GP, 2016, p. 1). Lo que plantea este nuevo paradigma de desarrollo es la necesidad de una apropiación más amplia de los beneficios, en términos de movilidad, flexibilidad e interactividad, del progreso en las TIC.

En la sociedad 5.0 -centrada en la persona, en el ser humano- la posibilidad de hacer uso de una gran cantidad de datos digítales para la provisión más eficiente de productos y servicios que satisfagan las distintas necesidades individuales debe traducirse, según los planteamientos realizados por el gobierno japonés, en nuevas ideas de innovación y negocios y en una reducción de las brechas sociales que aún existen en nuestras sociedades.

Mark Vidal plantea en su libro "La era de la humanidad" que la cuarta revolución es solamente el preámbulo de una quinta revolución de más alcance y hay que prepararse desde varios frentes: el económico, empresarial, social, educativo, y personal entre otros. Definir la industria 5.0 será definir la sociedad 5.0 , y son conceptos que no se pueden separar: estamos en camino a una sociedad totalmente automatizada, cognitiva y de accesos inmediatos donde todo funciona basado en robótica, inteligencia artificial, redes 5.G., por lo que el ser humano debe ser el objeto prioritario de esta sociedad 5.0. (Vidal, 2019). 
En ese sentido, el modelo de desarrollo que se propone para posibilitar la emergencia y sostenibilidad de la Sociedad 5.0 es la integración y conectividad inteligente entre ecosistemas que quizás, hasta el momento, han estado pensados como concernientes a sectores y dimensiones sociales diferentes. Sin embargo, esta necesidad de establecer vasos comunicantes entre distintos sectores y sistemas no es nueva.

El argentino Jorge Alberto Sábato propone en la década de los 60 , una estructura triangular entre la industria, la infraestructura científica y tecnológica y el estado como modelo para el desarrollo de la política de ciencia y tecnología en América Latina (Sábato, Botana 1968). En este modelo el estado, participa en el sistema como diseñador y ejecutor de la política. La infraestructura científico-tecnológica, como sector productor y oferente de la tecnología y el sector productivo, el cual es el demandante de tecnología. De la misma manera Vidal nos plantea que la tecnología debe servirnos para valorar más el factor humano y lograr ser más humanos a pesar de tener toda esta tecnología, ¿cómo ser más creativos, innovativos?

Una nueva sociedad está naciendo y lo hace con esta revolución "socio-tecnológica", los cambios tecnológicos crean oportunidades en la sociedad, hay que crear instituciones científicas y promover la relación universidad empresa.
La importancia de establecer articulaciones fuertes entre un sector generador de conocimiento y tecnología, un sector que se apropia de estos desarrollos y un sector que provee los incentivos adecuados y las políticas apropiadas para facilitar este intercambio, ha sido recurrente en varios modelos de desarrollo científico y tecnológico que siguieron las formulaciones de Sábato.

El sistema de innovación fue propuesto como un modelo funcional que motiva la innovación como el resultado de interacciones entre distintos tipos de actores (Lundvall, 1993); por su lado, el modelo de la triple hélice (Leydesdorff y Etzkowitz, 1993) resalta el papel de las organizaciones formales, y las relaciones entre los actores para que el conocimiento y la información incidan en el desarrollo económico y social de los países.

\section{En Colombia}

Aunque el término 5.0 se origina en parte como respuesta a un envejecimiento de la población japonesa, esta coyuntura ha dejado de ser una característica exclusiva de esta sociedad y de otras en países desarrollados. De acuerdo con los últimos datos censales para Colombia que presentó el DANE, el envejecimiento poblacional aplica también para nuestra sociedad colombiana. El índice de envejecimiento según el Censo Nacional de Población y Vivienda se ha incrementado 
Gráfica 1. Índice de envejecimiento en Colombia

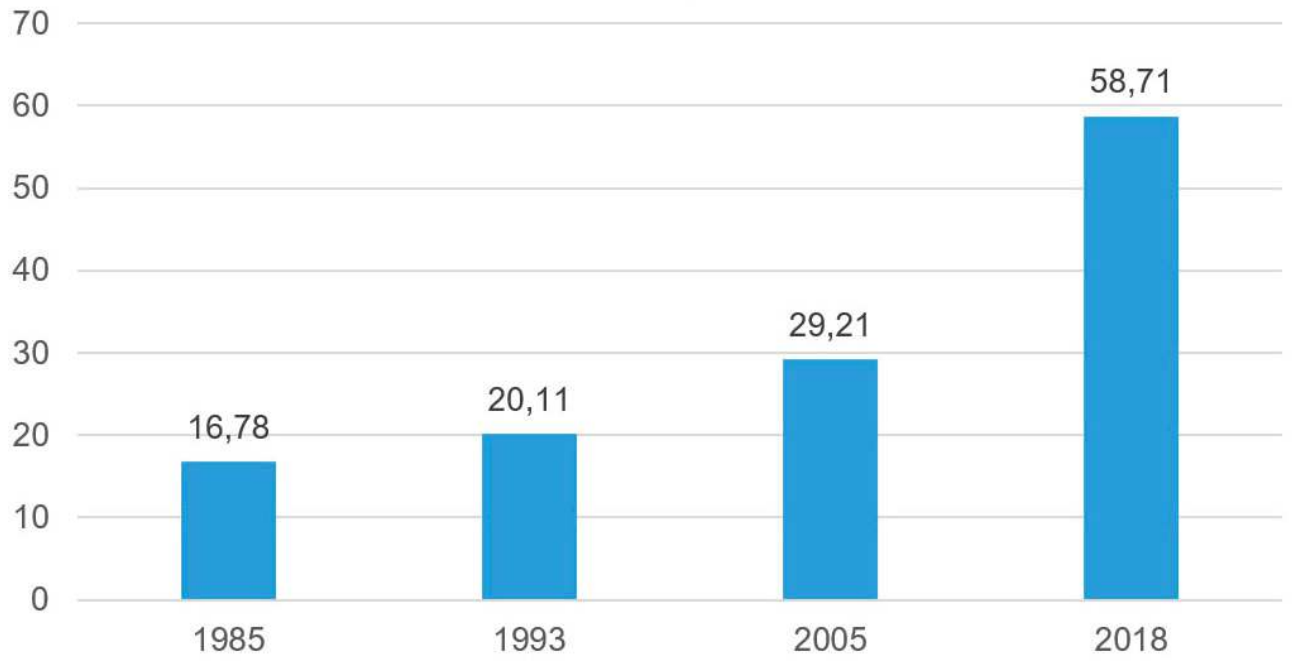

Gráfica 1. Fuente: Dane, 2018

en casi 30 puntos porcentuales en menos de 15 años, gráfica 1 .

Esto tiene algunas implicaciones en términos de la demanda por educación superior en el país. En el pasado número sobre transformación digital de esta revista (número 146), Luis Emilio Linares invitaba a reflexionar en los desafíos que la era digital acarreaban para quienes carezcan de las herramientas para participar en las nuevas oportunidades de negocio que la transformación digital invita, y la necesidad de revertir temores ante las nuevas tecnologías.

Es urgente procurar una educación en diferentes niveles como lo propone Jeimy J. Cano Martínez en el número sobre el analfabetismo de datos y su realidad (152). Es indispensable que los profesionales desarrollen competencias para la gestión y tratamiento de datos, aún más teniendo en cuenta que, de acuerdo con los resultados de la encuesta presentada en ese mismo número, casi el $60 \%$ de los profesionales de tecnología consideran que los colegios y las Instituciones de Educación Superior (IES) no están realizando los esfuerzos necesarios para la consolidación de competencias digitales en los estudiantes (Cano Martínez, 2018).

Las universidades deben pensar en el alfabetismo funcional propuesto en dicho número. En el diseño de procesos formativos que permitan el desarrollo de competencias no sólo para el trabajo, sino también para el consumo cultural, la adaptación a entornos en constante cambio, la apropiación de conceptos básicos, la interacción con nuestro entorno y con los otros, el desarrollo social y personal: 
competencias para satisfacer, de la manera más eficiente posible, las necesidades de cada individuo; esto permite "la realización del hombre en su propio contexto" (Cano Martínez, 2018) como se propone en el número. Cualquier modelo educativo que quiera superar este momento histórico debe ver este cambio como una oportunidad para liderar un cambio socio económico, cultural, tecnológico, un cambio en la forma de enseñar y aprender.

Pero además de este llamado a la educación superior, es importante también que en el país se concreten iniciativas para facilitar la conexión entre ecosistemas diversos de manera que se alcance un mayor valor agregado para nuestros productos y servicios (Linares, 2019). Las alianzas entre industria, academia y gobierno permitirán también un mayor impacto de los conocimientos generados, una apropiación de estos en función de las ne- cesidades y problemas del sector productivo y de la sociedad en general, y una mayor movilidad de investigadores entre organizaciones, sectores y disciplinas. En este sentido, es pertinente fomentar la colaboración universidad-empresa en los procesos de generación de conocimientos.

La gráfica 2, muestra la participación de las publicaciones en colaboración universidad-empresa dentro del total de publicaciones científicas de algunos países y regiones seleccionadas. En países como Japón, la articulación de los ecosistemas se puede evidenciar en la cantidad de publicaciones (casi un 6\%) que resultan de investigaciones en donde la universidad trabaja cerca al sector productivo en la solución de problemas relevantes para este a través de la generación de conocimientos. Colombia, con apenas 3\% de una producción científica significativa-

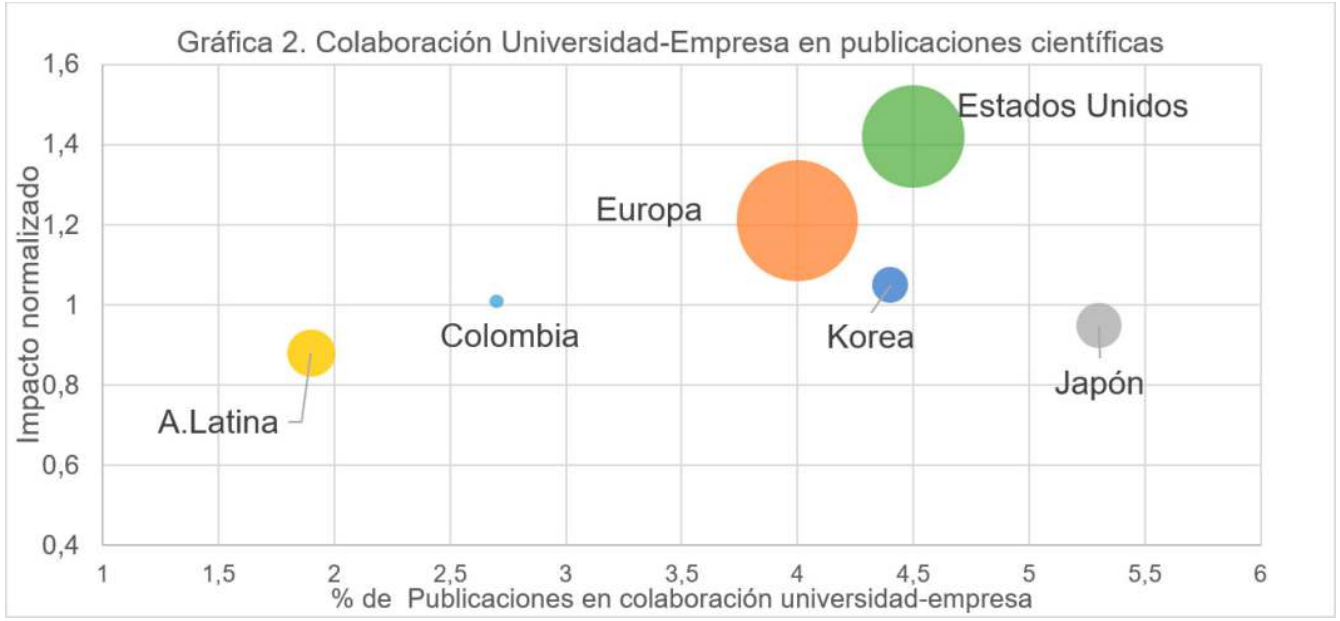

Gráfica 2. Fuente: SciVAL con datos de Scopus, 2020 
mente menor que Japón (representada en el tamaño del círculo), ha avanzado más que el promedio de la región en donde menos del $2 \%$ de las publicaciones reflejan este tipo de esfuerzo colaborativo.

\section{Conclusiones}

Las universidades deben reconocer su papel en la sociedad, como formadoras, pero también como generadoras de nuevos conocimientos. Las empresas colombianas, por su lado, deben reconocer el valor de la innovación como ventaja competitiva; tanto estas como el sector público, encuentran en las universidades un aliado para la innovación, tecnológica y productiva como innovación social, soluciones novedosas para las problemáticas sociales de nuestro país. La superposición de grupos de investigación y start-ups y estas intersectando con alianzas de grandes empresas, universidades y start-ups reflejan el surgimiento de patrones de relacionamiento entre la industria y la academia en campos como la biotecnología, ciencias de la computación entre otros. (Herrera, 2001). Estas serán la base de las empresas del futuro y hay que cambiar las estrategias empresariales que hoy se tienen.

Los grandes retos que propone el tránsito hacia una sociedad en donde el ser humano vuelva a ser el centro de los distintos modelos de desarrollo requiere no solo de importantes desarrollos en el campo de la tecnología, sino también de miradas críticas desde las ciencias sociales sobre las implicaciones en el desarrollo, sostenible e inclusivo, de las nuevas apuestas tecnológicas. Esto requiere un proceso de co-construcción participativa en el que, desde los distintos sectores, se reflexione sobre las deficiencias en educación, desarrollo de competencias, infraestructura o financiación y en las oportunidades que estas deficiencias ofrecen para el establecimiento de una sociedad centrada en el individuo, una sociedad 5.0. La tecnología que estimula la revolución económica e industrial no puede desvincularse del papel de la humanidad; de una sociedad que acumula pasaremos a una sociedad que utiliza; de la compra de productos pasaremos al uso de los servicios. El ser humano es el porqué y la tecnología es el cómo. (Vidal, 2019)

\section{Referencias}

Cano Martínez, J.J. (2018). Alfabetización de datos: Reflexiones iniciales sobre un saber necesario y emergente Jeimy J. Cano M. Sistemas, 152.

https://doi.org/10.29236/sistemas.n15 2a4

Dane (2018). Censo Nacional de Población y Vivienda 2018.

https://www.dane.gov.co/files/censo20 18/infografias/info-CNPC-2018totalnal-colombia.pdf

Government of Japan -GP, (2016), The 5th Science and Technology Basic Plan.

https://www8.cao.go.jp/cstp/english/ba sic/5thbasicplan.pdf 
Herrera, S. (2001) Academic research is the engine of Europe's biotech industry, Red Herring, 108, p.72-74.

Linares, L.E. (2019). La era digital: real desafío para la humanidad. Sistemas, 146.

https://doi.org/10.29236/sistemas.n14 $6 \mathrm{a} 2$

Leydesdorff, L. y Etzkowitz, H. (1996). Emergence of a Triple Helix of Univer- sity- Industry-Government Relations. Science and Public Policy, 23(5), 279286.

Sábato, J. A. y Botana, N. (1968). La ciencia y la tecnología en el desarrollo futuro de América Latina. Revista de la Integración, (3), 11.

Vidal, M. (2019). "La era de la humanidad" hacia la quinta revolución industrial, Ediciones Deusto.

Diana Lucio-Arias, PhD., Directora del Departamento de Ciencia de la Información de la Facultad de Comunicación y Lenguaje de la Pontificia Universidad Javeriana. Economista de la universidad Externado de Colombia, con Maestría en Información y Documentación de la universidad Carlos III de Madrid y Doctorado en Ciencias Sociales y del Comportamiento de la universidad de Ámsterdam en Holanda.

Maria Mercedes Corral Strassmann, MsC., Ingeniero de Sistemas y Computación de la universidad de Los Andes; Maestría en Comunicación de datos, University College London de la universidad de Londres; Programa de Desarrollo Directivo - PDD de Inalde. Experiencia, como Director de Proyectos en el Banco de la República; Gerente de TI de CIFIN Asobancaria; Vicepresidente de Tecnología de Deceval. Experiencia de más de 20 años como profesora universitaria en áreas de Ingeniería de software, y Gerencia de proyectos, Maestría y Especialización de Ingeniería de Sistemas en la Universidad Javeriana. Actualmente estudiante del Doctorado de Comunicación, Lenguajes e Información en la Universidad Javeriana. 